\title{
Preventive Analgesia Is Associated with Reduced Pain Disability 3 Weeks but Not 6 Montbs after Major Gynecologic Surgery by Laparotomy
}

Joel Katz, Ph.D., ${ }^{*}$ Lorenzo Cohen, Ph.D.†

Background: Most studies of preemptive or preventive analgesia restrict outcomes to pain and analgesic consumption in the acute postoperative period. The potential longer-term effects on these and other domains of functioning have received little empirical attention. The purpose of this study was to follow up patients who had received general anesthesia plus epidural fentanyl and lidocaine before (group 1) or after (group 2) incision or general anesthesia plus a sham epidural (group 3).

Methods: Patients were contacted approximately 3 weeks and 6 months after surgery. A follow-up pain questionnaire and the McGill Pain Questionnaire were administered by telephone. The Mental Health Inventory and Pain Disability Index were mailed to patients, completed, and mailed back.

Results: One hundred thirty-one of the 141 patients (93\%) were reached 3 weeks after surgery $(n=41, n=48$, and $n=42$ in groups 1, 2, and 3, respectively), and $109(77 \%)$ were reached at 6 months $(n=35, n=37$, and $n=37$ in groups 1,2 , and 3 , respectively). Multivariate analysis of covariance indicated that that even after controlling for age and presence or absence of preoperative pain, Pain Disability Index scores (mean \pm SD) at the first follow-up were significantly lower in group 1 (17.3 \pm 12.8) and group $2(18.1 \pm 17.0)$ compared with group $3(26.3 \pm$ 18.3). McGill Pain Questionnaire and Mental Health Inventory scores did not differ significantly among the groups. There were no significant differences at the 6-month follow-up.

Conchusion: The short-term beneficial effects of preventive epidural analgesia translated into less pain disability 3 weeks after surgery. Progress in understanding the processes involved in postsurgical recovery and the risk factors for chronic postsurgical pain would be aided by baseline and postsurgical measures of relevant psychological, emotional, and physical variables.

THE majority of patients who undergo surgery recover uneventfully and within weeks typically resume their normal daily activities. However, chronic postsurgical pain (CPSP) develops in an alarming proportion of patients. The magnitude of the problem is evidenced by

* Professor and Canada Research Chair, Department of Psychology and School of Kinesiology and Health Science, York University, Toronto, Ontario, Canada. Director, Acute Pain Research Unit, Department of Anesthesia and Pain Management, Toronto General Hospital and Mount Sinai Hospital, Toronto, Canada. Professor, Department of Anesthesia, University of Toronto, Ontario, Canada. $t$ Associate Professor, Department of Behavioral Science, The University of Texas, M. D. Anderson Cancer Center, Houston, Texas. recent studies that document the epidemiology and growing awareness of CPSP in the surgical community. ${ }^{1-6}$ For example, a prospective study of approximately 5,000 postsurgical patients estimated the incidence of acute neuropathic pain in the days after surgery to be between 1 and 3\%. ${ }^{7}$ Follow-up showed that 56\% continued to have ongoing pain $1 \mathrm{yr}$ later. This means that each year, between 0.05 and $1.5 \%$ of postsurgical patients continue to have pain $1 \mathrm{yr}$ after surgery. These statistics are staggering, especially when one considers the total number of patients worldwide who undergo surgery each year. It comes as no surprise then to see that almost $25 \%$ of more than 5,000 patients referred to chronic pain treatment centers have CPSP. ${ }^{8}$ Little is known about the biomedical and psychosocial factors that predict development of CPSP,9.10

One of the biomedical factors that has been linked to increased pain and analgesic consumption in the short and long term is the perioperative noxious injury barrage associated with surgery. ${ }^{11,12}$ Short-term reductions in pain and analgesic consumption have been reported in patients who received analgesic interventions designed to block or obtund the surgical injury barrage and subsequent noxious inputs from reaching the central nervous system. Most studies of preemptive or preventive analgesia ${ }^{11,12}$ have limited their outcomes to the assessment of pain and analgesic consumption in the acute postoperative period between the end of surgery and discharge from hospital. A series of studies has examined the effects of various perioperative analgesic regimens on postamputation phantom limb pain and stump pain 6 months to $1 \mathrm{yr}$ after patients have been discharged from hospital and resumed their daily activities. ${ }^{13}$ However, the longer-term effects of preemptive or preventive analgesia in patients undergoing other major surgical procedures has not received much empirical attention. ${ }^{14-20}$ With few exceptions, these studies have been limited to outcome measures of pain and analgesic consumption to the exclusion of other domains of functioning.

The purpose of the current study was to follow up patients who had participated in a randomized, doubleblind trial comparing postoperative pain and opioid consumption in patients who received general anesthesia plus epidural fentanyl and lidocaine before (group 1) or after (group 2) incision with a standard treatment control group (group 3) that received general anesthesia plus a sham epidural. ${ }^{21}$ In that study, pain on movement and secondary mechanical hyperalgesia $24 \mathrm{~h}$ after surgery, as well as cumulative morphine consumption at 
$48 \mathrm{~h}$, were reduced significantly in group 1 compared with group 3 . In addition, the hourly rate of morphine consumption between 24 and $48 \mathrm{~h}$ after surgery was significantly lower in group 1 compared with group 3 and in group 2 compared with group 3 . At the same time, the three groups were well matched in terms of demographics, clinical variables, mental health, mood, use of avoidant and active coping strategies, optimistic disposition, intrusive thoughts and avoidant behaviors, adjustment to stressful life events, and level of social support. The aim of the current study was to determine whether the three groups differed significantly in pain intensity and quality, pain disability, or general mental health 3 weeks and 6 months after surgery.

\section{Materials and Methods}

\section{Follow-up Interviews}

Ethics approval to perform the study was obtained from the University Health Network Research Ethics Board (Toronto, Ontario, Canada). All patients gave their written informed consent to participate before entering the study. Patients were contacted by telephone approximately 3 weeks and 6 months from the date of surgery. A maximum of five attempts was made to contact each patient by telephone. Neither the person conducting the interview nor the patient was aware of the group to which the patient had been assigned. A Follow-Up Pain Questionnaire and the McGill Pain Questionnaire (MPQ) were administered by telephone, and the following selfreport questionnaires were mailed to patients, completed, and mailed back to the researchers in a stamped, addressed, return envelope.

\section{Mental Health Inventory}

The Mental Health Inventory (MHI) ${ }^{22}$ is a self-administered questionnaire that measures symptoms of psychological distress and well-being. The current study used an 18-item version of the MHI that consists of a total score and five subscales: anxiety, depression, loss of behavioral/emotional control, positive affect, and interpersonal ties. ${ }^{23}$ Subjects responded to each of the 18 statements on the basis of how often in the past month they have experienced each symptom. Each statement is accompanied by a six-choice response set ranging from $1=$ all of the time to $6=$ none of the time. The total score, which we report in the current study, ranges from 0 to 108 , with higher scores indicative of better mental health. The MHI has adequate reliability and validity. ${ }^{22}$

\section{Pain Disability Index}

The Pain Disability Index (PDI) ${ }^{2 f}$ was developed to determine the extent to which pain interferes with seven daily, psychosocial activities and functions, including (1) family/home responsibilities, (2) recreation, (3) social activity, (4) occupation, (5) sexual behavior, (6) self-care, and (7) life-support activity. Each category is rated on an 11-point scale ranging from 0 (no disability) to 10 (total disability). The total score ranges from 0 to 70. The PDI has been shown to have high internal consistency $(0.86)$, modest test-retest reliability $(0.44)$, and adequate concurrent validity.

\section{McGill Pain Questionnaire}

The MPQ was developed by Melzack ${ }^{25}$ to obtain quantitative and qualitative measures of the experience of pain. The MPQ yields two global scores, the pain rating index and the present pain intensity, which have been found to provide valid and reliable measures of pain. ${ }^{25,26}$ The total pain rating index is the sum of the rank values of the words chosen from 20 sets of qualitative words, each set containing two to six adjectives that describe the sensory, affective and evaluative properties of pain. The lists of pain descriptors were read to the patients who were asked to choose the word in each category that best described their pain at the moment. The present pain intensity is rated on a scale of $0-5$ as follows: $0=$ none, $1=$ mild, $2=$ discomforting, $3=$ distressing, $4=$ horrible, and $5=$ excruciating. The MPQ was not administered to patients who did not report having pain at the time of the telephone interviews.

\section{Follow-up Pain Questionnaire}

The Follow-up Pain Questionnaire is a brief inventory designed to assess the presence, intensity, location, frequency, and quality of long-term postsurgical pain. Items also assess pain interference in daily life, methods of pain relief sought, medication use, and aggravating and relieving factors. The Follow-up Pain Questionnaire was modeled after similar pain assessment measures, including the $\mathrm{MPQ}^{25}$ and a follow-up interview form used to assess long-term pain after surgery. ${ }^{27}$

\section{Statistical Analyses}

Data were analyzed using the Statistical Package for the Social Sciences (SPSS for Windows, release 11.5.0; Chicago, IL). Incidence and frequency of pain at each follow-up ( 3 weeks and 6 months) were compared among groups by chi-square analysis. Pain intensity and quality and psychosocial data at each follow-up were analyzed by one-way multivariate analysis of covariance using group (1,2, and 3$)$ as the independent samples factor; age and preoperative pain status as the covariates; and MHI total score, MPQ total pain rating index, and PDI total score as the dependent variables. A significant multivariate effect of group was followed up with separate univariate analysis of covariance on each of the three dependent variables. A significant univariate effect of group was follow-up with post boc tests using the least significant difference method to determine the pattern 
Table 1. Scores on Measures of Pain, Pain Disability, and Mental Health at the First Follow up

\begin{tabular}{lccc}
\hline & Group 1 (Before Incision) & Group 2 (After Incision) & Group 3 (Control) \\
\hline Days between surgery and interview & $22 \pm 8.2$ & $25 \pm 11.1$ & $24 \pm 9.4$ \\
Worst pain since discharge (0-10) & $5.2 \pm 2.6$ & $4.9 \pm 2.7$ & .3 \\
McGill Pain Questionnaire* & & & $5.3 \pm 2.5$ \\
Pain rating index-total & $12.9 \pm 10.9$ & $15.9 \pm 12.0$ & $1.1 \pm 0.8$ \\
$\quad$ Current pain intensity & $1.3 \pm 0.9$ & $9.2 \pm 7.0$ & $1.2 \pm 1.0$ \\
Number of words chosen & $8.7 \pm 6.2$ & $18.1 \pm 17.0$ & $9.2 \pm 8.7$ \\
Pain Disability Indext & $17.3 \pm 12.8$ & $89.8 \pm 14.6$ & $26.3 \pm 18.3$ \\
Mental Health Inventory-18 & $89.3 \pm 9.6$ & $89.9 \pm 11.9$ \\
\hline
\end{tabular}

Data are presented as mean $\pm S D$.

*McGill Pain Questionnaire scores are from patients who reported being in pain at the time of the interview $(n=28, n=33, n=33$ for groups 1,2, and 3, respectively). $\quad+P<0.03$ for group 1 vs. group 3 and group 2 vs. group 3.

of significance among the three means. The separate domains of pain disability at the 3-week follow-up were analyzed by one-way multivariate analysis of variance. $P$ $<0.05$ was considered significant.

\section{Results}

\section{First Follow-up Interview}

In total, 131 of the 141 patients (93\%) were reached by telephone approximately 3 weeks after surgery, were interviewed, and subsequently mailed back the completed questionnaires $(n=41, n=48$, and $n=42$ in groups 1,2 , and 3 , respectively). The overall incidence of pain among patients who were contacted was $75.6 \%$, with no significant differences among the three groups $(n=30[73.2 \%], n=34[70.8 \%]$, and $n=35[83.3 \%]$ for groups 1,2 , and 3 , respectively).

The pain was mild to discomforting among patients who reported being in pain at the time of the interview (table 1). Approximately $30 \%$ of all patients were taking analgesic tablets on a regular basis $(\mathrm{n}=13, \mathrm{n}=11$, and $\mathbf{n}=11$ for groups 1, 2, and 3, respectively). Analgesics included nonsteroidal antiinflammatory drugs alone $(\mathrm{n}=$ 5) or with acetaminophen $(\mathbf{n}=2)$, acetaminophen alone $(n=9)$ or with codeine $(n=15)$, acetaminophen and amitriptyline $(\mathrm{n}=1)$, oxycodone and acetylsalicylic acid/acetaminophen $(n=2)$, and pentazocine $(n=1)$. The three groups did not differ significantly in pain frequency (table 2).

A one-way between-groups multivariate analysis of covariance on MPQ total pain rating index, MHI, and PDI scores using age and presence or absence of preoperative pain as covariates revealed a significant multivariate effect of group and the two covariates (all $P<0.04$ by Roy's largest root). Separate one-way univariate analyses of covariance on the three dependent variables showed a significant group effect for PDI scores $(P=0.011)$ and the covariate preoperative pain status $(P<0.0009)$, indicating that even after controlling for age and presence or absence of preoperative pain, pain disability at the first follow-up was lower in groups 1 and 2 compared with group 3. Post boc tests using the least significant difference approach showed that patients in group 3 reported significantly more pain-related disability than patients in group 1 and group 2 (both $P<0.03$ ). PDI scores in group 1 did not differ significantly from those in group 2 (table 1). A multivariate analysis of variance on the specific domains of pain disability was not significant (table 3). There were no other significant differences among the groups at the first follow up.

\section{Second Follow-up Interview}

In total, 109 of the 141 patients (77\%) were reached by telephone 6 months after surgery, were interviewed, and

Table 2. Number of Patients Reporting Various Pain Frequencies and Aggravating Factors at the First Follow up

\begin{tabular}{lrrr}
\hline & Group 1 (Before incision) & Group 2 (After incision) & Group 3 (Control) \\
\hline Pain frequency & & & \\
Constant (continuous, steady) & $6(14.6)$ & $2(4.2)$ & $5(11.9)$ \\
Periodic (rhythmic, intermittent) & $14(34.2)$ & $18(37.5)$ & $18(42.9)$ \\
$\quad$ Brief (momentary, transient) & $9(21.9)$ & $14(29.2)$ & $12(28.6)$ \\
Aggravating factors & & & \\
Touching scar & $12(29.3)$ & $13(27.1)$ & $11(26.2)$ \\
Sitting up from lying position & $20(48.8)$ & $24(50.0)$ & $16(38.1)$ \\
Walking & $15(36.6)$ & $19(39.6)$ & $14(33.3)$ \\
Taking a deep breath & $5(12.2)$ & $2(4.2)$ & $3(7.1)$ \\
Emotional stress & $7(17.3)$ & $2(4.2)$ & $7(14.6)$ \\
Carrying heavy objects & $10(24.4)$ & $27(56.3)$ & $13(30.9)$ \\
Coughing & $20(48.8)$ & & $24(57.1)$ \\
\hline
\end{tabular}

Data are presented as incidence $(\%)$. 
Table 3. Scores on the Seven Categories of the Pain Disability Index at the First Follow up after Surgery

\begin{tabular}{lcrr}
\hline \multicolumn{1}{c}{ Pain Disability Index Categories } & Group 1 (Before incision) & Group 2 (After incision) & Group 3 (Control) \\
\hline Family/home responsibilities & $2.85 \pm 2.5$ & $2.97 \pm 2.9$ & $3.97 \pm 3.2$ \\
Recreation & $2.79 \pm 2.5$ & $3.32 \pm 3.5$ & $4.78 \pm 3.3$ \\
Social activity & $2.03 \pm 2.5$ & $2.48 \pm 3.2$ & $3.47 \pm 3.2$ \\
Occupation & $3.14 \pm 2.7$ & $3.46 \pm 3.7$ & $-1.02 \pm 3.8$ \\
Sexual activity & $4.83 \pm 3.5$ & $4.02 \pm 3.8$ & $5.8 \pm 3.8$ \\
Self-care & $0.88 \pm 1.7$ & $1.08 \pm 1.7$ & $1.89 \pm 2.5$ \\
Life support activity & $0.74 \pm 1.9$ & $0.74 \pm 1.5$ & $1.39 \pm 2.1$ \\
\hline
\end{tabular}

Data are presented as mean \pm so. In contrast to the total Pain Disability Index score shown in table 1 , differences among the groups in the individual Pain Disability Index categories are not statistically significant by multivariate analysis of variance.

subsequently mailed back the completed questionnaires $(\mathrm{n}=35, \mathrm{n}=37$, and $\mathrm{n}=37$ in groups 1,2 , and 3 , respectively). Because $23 \%$ of the patients were lost to follow-up, a random sample of the patients who were reached 6 months after surgery was generated using SPSS by matching them on the group factor to patients who were lost to follow-up. The two subgroups were then compared on demographic, baseline clinical and psychosocial variables, and postoperative pain scores and morphine consumption. There were no significant differences on any variable, suggesting that patients who were lost to follow-up did not differ significantly from those who were reached 6 months after surgery.

The overall incidence of pain among patients contacted was $32.1 \%$, with no significant difference among the three groups $(31.4 \%[\mathrm{n}=11], 29.7 \%[\mathrm{n}=11]$, and $35.1 \%[\mathrm{n}=$ 13] for groups 1,2 , and 3 , respectively). Four patients (one in group 1 and three in group 3) who reported that they did not have pain at 4 weeks reported pain at 6 months.

The pain was rated as mild among patients who reported being in pain at the time of the interview (table 4). Only three patients reported taking analgesic medication for the pain ( $n=2$ in group 1 and $n=1$ in group 2). There were no significant differences among the groups in pain, pain disability, or mental health at the 6-month follow-up.

\section{Discussion}

The results of the current study suggest that the shortterm beneficial effects of preventive epidural analgesia (i.e., given before or after incision) on acute postoperative pain and morphine consumption are associated with lower levels of pain disability approximately 3 weeks after surgery, even after controlling for age and preoperative pain history. PDI scores in the two groups that received general anesthesia plus epidural lidocaine and fentanyl were significantly lower 3 weeks after surgery than those of the control group, which received general anesthesia alone. The absence of concomitant pain intensity differences at the 3-week follow-up may indicate that the reduced hyperalgesia (group 1) and rate of morphine consumption (groups 1 and 2) within the first 2 days after surgery afforded the epidural groups a "head start" in terms of comfort level and recovery compared with the control group. A similar finding has been reported with respect to activity levels 3.5 weeks after radical retropubic prostatectomy. ${ }^{16}$ In that study, activity levels but not pain intensity were significantly higher in patients who had received preemptive epidural bupivacaine or fentanyl.

Our data provide further empirical support for a distinction between the narrow definition of preemptive analgesia and the broader concept of preventive analgesia. ${ }^{11,28}$ Preemptive analgesia requires that a preoperative analgesic intervention reduce pain or analgesic consumption to a greater extent than the identical intervention administered after incision or surgery. In contrast, the aim of preventive analgesia is to minimize sensitization arising from preoperative, intraoperative, and/or postoperative noxious stimuli; evidence for preventive analgesia does not require inclusion of a preop-

Table 4. Scores on Measures of Pain, Pain Disability, and Mental Health at the Second Follow up

\begin{tabular}{lccc}
\hline & Group 1 (Before incision) & Group 2 (After incision) & Group 3 (Control) \\
\hline Days between surgery and interview & $187 \pm 12.0$ & $191 \pm 16.4$ & $187 \pm 11.0$ \\
Worst pain since discharge (0-10) & $4.6 \pm 2.6$ & $4.3 \pm 2.7$ & $5.7 \pm 2.5$ \\
McGill Pain Questionnaire* & & & $7.2 \pm 6.7$ \\
Pain rating index-Total & $12.1 \pm 12.0$ & $0.2 \pm 0.6$ & $7.0 \pm 7.2$ \\
Current pain intensity & $0.4 \pm 0.8$ & $3.9 \pm 3.3$ & $0.5 \pm 0.8$ \\
Number of words chosen & $7.4 \pm 8.2$ & $3.3 \pm 7.0$ & $4.3 \pm 4.1$ \\
Pain Disability Index & $4.6 \pm 8.1$ & $86.2 \pm 15.2$ & $3.6 \pm 6.1$ \\
Mental Health Inventory-18 & $86.9 \pm 15.7$ & & $88.2 \pm 14.0$ \\
\hline
\end{tabular}

Data are presented as mean \pm SD.

${ }^{*}$ McGill Pain Questionnaire scores are from patients who reported being in pain at the time of the interview $(\mathrm{n}=9, \mathrm{n}=13, \mathrm{n}=11$ for groups 1,2 , and 3 , respectively). 
erative analgesic intervention. Therefore, the finding that postincisional epidural analgesia resulted in reduced pain disability compared with the control group is evidence of a preventive effect. These results argue for the inclusion of a clinically relevant control group that receives standard care in addition to the two-group design typically used in studies of preemptive analgesia. In the current study, inclusion of a standard treatment control group allowed us to detect differences in pain disability 3 weeks after surgery that would have gone undetected had we used the two-group design typically used in studies of preemptive analgesia (i.e., preoperative $v s$. postoperative analgesic administration).

We do not know the mechanisms by which the early preventive effect of the epidural regimen translates into reduced pain disability 3 weeks later. It is possible that the differences in morphine consumption and pain on movement observed early on after surgery led to increased self-efficacy for postsurgical ambulation ${ }^{29}$ and decreased fear-avoidance behaviors ${ }^{30,31}$ in the treated groups. The hypothesized head start would have enabled the treated groups to resume activities of daily life earlier than the control group, leading to lower pain disability at 3 weeks in the treated groups compared with the control group in the face of nonsignificant group differences in pain intensity and quality. Another related possibility is that the 3 -week pain disability reduction in groups 1 and 2 is due to a reduction in 3-week movement pain, which we did not directly assess because many of the items in the PDI include activities involving physical movement. The data in table 2 describing aggravating factors (e.g., walking, coughing, and sitting up from a lying position), however, do not support this suggestion, at least as it relates to group differences in the incidence of activities that generate pain, but it may be that differences exist in the intensity of pain associated with these activities. A third possibility is that the greater rate of patient-controlled analgesia morphine consumption after surgery in group 3 was associated with opioid-induced facilitation of nociceptive processing ${ }^{32,33}$ that, in some as yet unspecified way, contributed to increased pain disability 3 weeks after surgery. These possibilities remain speculative and require empirical validation by expanding the narrow range of outcome variables typically assessed in studies of analgesic efficacy to include relevant psychosocial and physical factors. ${ }^{34}$

By 6 months after surgery, the three groups were indistinguishable in terms of all measured outcomes. The differences observed at 3 weeks were no longer apparent, and psychosocial measures of mental health and pain disability were in the normal range. Although other studies have evaluated the effects on long-term pain of blocking noxious afferent processing perioperatively, only one other has extended the follow-up assessment to include non-pain-related, psychosocial variables. ${ }^{20}$ In that study, the acute postoperative opioid-sparing effects of the perioperative epidural regimen in men undergoing radical prostatectomy was not associated with group differences in pain incidence, intensity, or quality of life at a 3- or 6-month follow-up assessment. Nevertheless, independent of the perioperative analgesic regimen, the overall incidences of CPSP were $49 \%$ and $35 \%$, respectively, at the two follow-ups ${ }^{20}$ Although the pain was generally mild in intensity, patients reported reduced physical and social functioning as well as poor overall health 3 and 6 months after surgery. In the current study, the incidence of pain 6 months after surgery was approximately $32 \%$. In general, pain intensity was mild, and $10 \%$ of the patients were taking analgesic medication. These results are consistent with past research on a similar patient population ${ }^{15}$ in which the incidence of pain at 6 months experienced within the previous week was $40 \%$, although the intensity was greater than in the current study.

There are limitations to the current study. First, patients were not examined physically but were interviewed over the telephone. Therefore, we have little basis on which to determine the nature of the mechanisms underlying the observed differences in pain disability between treated and control groups. Second, patients with persistent pain were not actively seeking treatment but had been contacted as a follow-up to the original study. These patients come from a different population that those with postsurgical pain, who are referred to specialty pain clinics, ${ }^{8}$ and therefore, generalization to patients with chronic pain is not warranted. Third, we documented presence or absence of preoperative pain at the preadmission visit before surgery but did not obtain a baseline measure of pain disability using the PDI. Although these two variables are clearly related and the difference in pain disability among the groups at the first follow-up was significant after controlling for preoperative pain, we do not know whether this effect would have been found had we controlled for preoperative pain disability using the PDI. Finally, we cannot rule out the possibility that a bias may have been introduced to the 6-month assessment data because $23 \%$ of the patients were lost to follow-up. However, we did not find significant differences on a variety of demographic, psychosocial, and pain-related variables between a random sample of patients who were reached and those who were lost to follow-up 6-months after surgery.

In summary, pain disability 3 weeks after abdominal gynecologic surgery by laparotomy was significantly lower among patients who had received general anesthesia plus perioperative lumbar epidural than among those who underwent surgery during general anesthesia alone, providing evidence for an extended benefit associated with preventive analgesia. The advantage conferred by preventive analgesia was no longer evident at a 6-month follow-up. Preoperative blockade followed by 
prolonged blockade of noxious inputs well into the postoperative period may prove to be the most effective way of managing acute postoperative pain and possibly preventing development of pain-related disability. Given the prominent role of psychosocial factors in chronic pain $^{35}$ and the recent recommendation for assessment of core domains in clinical trials, ${ }^{34}$ we suggest that future studies include an assessment at baseline and after surgery of relevant psychological, emotional, and physical variables in addition to the standard biomedical factors (i.e., pain and analgesic consumption) typically measured. Inclusion of these variables may help to shed light on the processes involved in recovery from surgery and the risk factors for developing CPSP.

The authors thank Tanya Constantine, B.Sc.N., R.N. (Clinical Trials Nurse, Division of Clinical Trials and Epidemiology, Toronto Sunnybrook Regional Cancer Centre, Toronto, Ontario, Canada); Mariel Escover, R.N., B.Sc.N. (Clinical Retina Research Coordinator, Department of Vision Science Research, Toronto Western Hospital); Olivera Karanovic, M.D. (Research Coordinator, Department of Vision Science Research, Toronto Western Hospital, Toronto, Ontario, Canada): Keitha McMurray, B.Sc.N., R.N., M.Sc. (Process Coordinator, Division of Clinical Trials and Epidemiology, Totonto Sunnybrook Regional Cancer Centre) and Adarose Wowk, B.Sc., R.N. (Clinical Trials Monitor, Aventis Pharma, Toronto, Ontario, Canada), for their expertise with patient interviews and help in data scoring, entry, and verification.

\section{References}

1. Poobalan AS, Bruce J, Smith WC, King PM, Krukowski ZH, Chambers WA A review of chronic pain after inguinal herniorrhaphy. Clin J Pain 2003; 19:48-54 2. Perttunen $K$, Tasmuth T, Kalso E: Chronic pain after thoracic surgery: $A$ follow-up study. Acta Anaesthesiol Scand 1999; 43:563-7

3. Passlick $B$, Born $C_{2}$ Sienel $W$, Thetter $O$ : Incidence of chronic pain after minimal-invasive surgery for spontaneous pneumothorax. Eur $\mathrm{J}$ Cardiothorac Surg 2001; 19: 355-8

4. Bruce J, Drury N, Poobalan AS, Jeffrey RR, Smith WC, Chambers WA: The prevalence of chronic chest and leg pain following cardiac surgery: A historical cohort study. Pain 2003; 104:265-73

5. Kalso E, Mennander S, Tasmuth T, Nilsson E: Chronic post-sternotomy pain Acta Anaesthesiol Scand 2001; 45:935-9

6. Macrae WA: Chronic pain after surgery. Br J Anaesth 2001; 87:88-98

7. Hayes $C$, Browne $S$, Lantry $G$, Burstal $R$ : Neuropathic pain in the acute pain service: A prospective study. Acute Pain 2002; $4: 45-8$

8. Crombie IK, Davies HT, Macrae WA: Cut and thrust: antecedent surgery and trauma among patients attending a chronic pain clinic. Pain 1998; 76:167-71

9. Perkins FM, Kehlet H: Chronic pain as an outcome of surgery; A review of predictive factors. ANESTHESIOLOGY 2000; 93:1123-33

10. Katz J: Peri-operative predictors of long-term pain following surgery Proceedings of the 8th World Congress on Pain, Progress in Pain Research and Management. Edited by Jensen TS, Turner JA, Wiesenfeld-Hallin Z. Seattle, IASP Press, 1997, pp 231-40

11. Katz $\mathrm{J}$ : Timing of treatment and pre-emptive analgesia, Clinical Pain Man agement: Acute Volume. Edited by Rice A, Warfield C, Justins D, Eccleston C. London, Arnold, 2003, pp 113-62

12. McCartney ClL, Sinha A, Katz J: Systematic review of the role of the
$\mathrm{N}$-methyl D-aspartate (NMDA) receptor antagonists in preventive analgesia. Anesth Analg 2004; 98:1385-400

13. Katz J: Phantom limb pain. Lancet 1997; 350:1338-9

14. Katz J, Jackson M, Kavanagh BP, Sandler AN: Acute pain after thoracic surgery predicts long-term post-thoracotomy pain. Clin J Pain 1996; 12:50-5

15. Katz J, Clairoux $M$, Redahan $C$, Kavanagh BP, Carroll $S$, Nierenberg $H$ Jackson $M$, Beattie J, Taddio A, Sandler AN: High dose alfentanil pre-empts pain after abdominal hysterectomy. Pain 1996; 68:109-18

16 Gottschalk A, Smith DS, Jobes DR, Kennedy SK, Lally SE, Noble VE Grugan KF, Seifert HA, Cheung A, Malkowicz SB, Gutsche BB, Wein AJ: Preemptive epidural analgesia and recovery from radical prostatectomy: A randomized controlled trial. JAMA 1998; 279:1076-82

17. Gundes H, Kilickan L, Gurkan Y, Sarlak A, Toker K: Short- and long-term effects of regional application of morphine and bupivacaine on the iliac crest donor site. Acta Orthop Belg 2000; 66:341-4

18. Obata H, Saito S, Fujita N, Fuse Y, Ishizaki K, Goto F: Epidural block with mepivacaine before surgery reduces long-term post-thoracotomy pain. Can J Anaesth 1999: 46:1127-32

19. Reuben SS, Vieira P, Faruqi S, Verghis A, Kilaru PA, Maciolek H: Local administration of morphine for analgesia after iliac bone graft harvest. ANESTHEStOLOGY 2001; 95:390-4

20. Haythornthwaite JA, Raja SN, Fisher B, Frank SM, Brendler CB, Shir Y: Pain and quality of life following radical retropubic prostatectomy. J Urol 1998; 160:1761-4

21. Katz J, Cohen L, Schmid R, Chan VW, Wowk A: Postoperative morphine use and hyperalgesia are reduced by preoperative but not intraoperative epidural analgesia: Implications for preemptive analgesia and the prevention of central sensitization. AnEsTHESIOLOGY 2003; 98:1449-60

22. Ware JEJ, Johnston SA, Davies-Avery A, Brook RH: Conceptualization and Measurement of Health for Adults in the Health Insurance Study. Vol III. Mental Health (R-1987/3-HEW). Santa Monica, RAND Corporation, 1979

23. Weinstein MC, Berwick DM, Goldman PA, Murphy JM, Barsky A: A comparison of three psychiatric screening tests using receiver operating characteristics (ROC) analysis. Med Care 1989; 27:593-607

24. Tait RC, Chibnall JT, Krause S: The Pain Disability Index: Psychometric properties. Pain 1990; 40:171-82

25. Melzack R: The McGill Pain Questionnaire: Major properties and scoring methods. Pain 1975; 1:277-99

26. Katz J, Melzack R: Measurement of pain. Surg Clin North Am 1999; $79: 231-52$

27. Dajczman E, Gordon A, Kriesman H, Wolklove N: Long-term posthoracotomy pain. Chest $1991 ; 99: 270-4$

28. Kissin I: Preemptive analgesia: terminology and clinical relevance (letter; comment). Anesth Analg 1994; 79:809-10

29. Pellino T, Thuczek A, Collins M, Trimborn S, Norwick H, Engelke ZK, Broad J: Increasing self-efficacy through empowerment: Preoperative education for orthopaedic patients. Orthopaedic Nursing 1998; 17:48-51, 54-9

30. Philips $\mathrm{HC}$ : Avoidance behaviour and its role in sustaining chronic pain. Behav Res Ther 1987: 25:273-9

31. Vlaeyen JWS, Linton SJ: Pear-avoidance and its consequences in chronic musculoskeletal pain: State of the art. Pain 2000; 85:317-32

32. Celerier E, Laulin J, Larcher A, Le Moal M, Simonnet G: Evidence for opiate-activated NMDA processes masking opiate analgesia in rats. Brain Res $1999 ; 847: 18-25$

33. Li X, Angst MS, Clark JD: Opioid-induced hyperalgesia and incisional pain. Anesth Analg 2001; 93:204-9

34. Turk DC, Dworkin RH, Allen RR, Bellamy N, Brandenburg N, Carr DB, Cleeland C, Dionne R, Farrar JT, Galer BS, Hewitt DJ, Jadad AR, Katz NP, Kramer LD, Manning DC, McCormick CG, McDermott MP, McGrath P, Quessy S, Rappaport BA, Robinson JP, Royal MA, Simon L, Stauffer JW, Stein W, Tollett J, Witter J: Core outcomes for chronic pain clinical trials: IMMPACT recommendations. Pain $2003 ; 106: 337-45$

35. Turk DC: Cognitive-behavioral approach to the treatment of chronic pain patients. Reg Anesth Pain Med 2003; 28:573-9 